( $n=5$ ). Non-target lipid levels were detected in $44.7 \%$ of low-risk patients, in $83 \%$ of medium risk patients, in $78.6 \%$ of high risk patients, in $100 \%$ of very high risk patient. Absolute indications for statin therapy were detected in $17.4 \%$.

Hemodynamically insignificant carotid plaques (stenosis $<50 \%$ ) was detected in $56.5 \%$ of patients with RA: in $23.7 \%$ of low risk category, in $74.3 \%$ of medium risk patients, in $85.7 \%$ of high risk patients, in $100 \%$ of very high risk patients. A sever calcification of the coronary arteries was detected in $25 \%$ (the coronary index is more than $300 \mathrm{U}$ or $>75$ percentile of the age- and sex-related reference values, in according to MESA): in $5.3 \%$ of low risk patients, in $37 \%$ of medium risk patients, in $28.6 \%$ of high risk patients, in $80 \%$ of very high risk.

After carotid ultrasound $38 \%(34 / 92)$ of RA patients were reclassified from the category of low and medium cardiovascular risk on the mSCORE scale to the high risk category. The proportion of patients with high cardiovascular risk increased in 3.5 times $(53.2 \%, p<0.001)$. There were 42 patients with non-target lipid levels in the high risk category. The proportion of patients with absolute indications for statins increased in 2.9 times compared with the results of the mSCORE evaluation ( $\mathrm{n}=$ 47/92; 51\%; p <0.001).

After MDCT assessment of coronary calcification $16.3 \%(15 / 92)$ of RA patients were reclassified from the category of low and medium cardiovascular risk on the mSCORE scale to the high risk category. The proportion of patients with high cardiovascular risk increased in 2.1 times $(31.5 \%, p<0.001)$. There were 24 patients with non-target lipid levels in the high risk category. The proportion of patients with indications for statins increased in 1.8 times compared with the results of the mSCORE evaluation ( $n=29 / 92 ; 31.5 \% ; p<0.001$ ). Carotid ultrasound more often reveals indications for statin therapy, $\mathrm{p}<0.001$.

Conclusion: The use of the mSCORE scale is not sensitive enough for stratification of cardiovascular risk in RA patients due to the high incidence of subclinical atherosclerosis in this population. The use of carotid ultrasound and the MDCT assessment of coronary calcification significantly improve the stratification of cardiovascular risk in patients with RA, which helps to identify patients requiring intensive prevention of cardiovascular complications. The frequency of identifying patients with absolute indications for statin therapy when performing carotid ultrasound is significantly higher than MDCT assessment of coronary calcification.

Disclosure of Interests: None declared

DOI: 10.1136/annrheumdis-2019-eular.3971

\section{AB0356 COMPARISION FOREARM BONE MINERAL DENSITY BETWEEN LUMBAR SPINE AND HIP: A USEFUL TOOL TO SCREEN OSTEOPOROSIS IN FEMALE PATIENTS WITH RHEUMATOID ARTHRITIS}

\section{Yu Wang, Zhuoli Zhang. First hospital of Peking University, Beijing, China}

Background: DXA is widely used in clinical practise in BMD measurement of osteoporosis. Forearm and axial BMD is the most common site to define in clinical practise. However, the relationship between forearm and axial BMD is not confirmed in RA patients.

Objectives: To compare the forearm (bone mineral density) BMD between lumbar spine and left hip BMD by dual-energy X-ray abosorptionmetry (DXA), and explore the diagnostic value of the forearm BMD in rheumatoid arthritis (RA) patients.

Methods: In the study, 200 female patients with established RA underwent DXA of the lumber, left hip and non-superiority forearm DXA at the same time. We compared BMD at different sites, and the diagnostic cutoff value of abnormal axial BMD by forearm BMD was explored. Data analysis was performed by independent-sample $t$ test and Pearson's correlation test. The area under the receiver operating characteristic curve (AUC) and calibration of predictions were assessed. Sensitivity and specificity were calculated to determine the correlation between cases of osteoporosis detected by the axial DXA scan and forearm.

Results: (1) The mean age of the 200 female patients was $(55.9 \pm 13.8)$ years. Twenty $(10.0 \%)$ patients had the fragility fracture history. Based on their axial DXA data and fracture history, 30 (15.0\%) patients had normal BMD (T score $\geq-1.0), 170(85.0 \%)$ patients had abnormal BMD. The mean forearm BMD was $(0.35 \pm 0.13) \mathrm{g} / \mathrm{cm} 2$, and $T$ score was $-2.3 \pm 1.9$. (2) Compared with abnormal axial BMD group, forearm BMD in normal axial group was significantly decreased $[(0.33 \pm 0.13) \mathrm{g} / \mathrm{cm} 2$ vs $(0.44 \pm 0.06)$ $\mathrm{g} / \mathrm{cm} 2, \mathrm{t}=4.29, \quad \mathrm{P}<0.01]$, forearm $\mathrm{T}$ score was also significantly decreased $[(-2.6 \pm 1.9)$ vs $(-0.71 \pm 0.9), t=4.35, \quad P<0.01]$. T score of the forearm BMD was positively correlated with axial BMD $T$ score, both at lumber and left hip respectively $(r=0.488,0.543, P<0.01)$. After correction by age, $\mathrm{T}$ score of the forearm remained positively correlated with axial $T$ score by partial correlation analysis (Pearson partial vertebral $r=0.46, \quad P<0.001$; left hip $r=0.502$, $P<0.001)$. (3) The area under the ROC curve for diagnosis of lumber and hip osteoporosis were $0.757(95 \% \mathrm{Cl}: 0.638,0.876)$ and 0.615 (95\% $\%$ : $0.481,0.749)$ separately. (4) The sensitivity and specificity for identifying osteoporosis in lumber were $86.5 \%$ and $50.1 \%$ respectively when the T-score threshold of forearm was defined as -3.52; however, the sensitivity and the specificity for identifying osteoporosis at left hip were $66.2 \%$ and $76.2 \%$ when $\mathrm{T}$ score threshold of forearm was defined as -2.5 .

Conclusion: Our study has confirmed that DXA measurement performed of forearm analysis is capable of screening osteoporosis defined by axia BMD in female RA patients.

\section{REFERENCES}

[1] Siris ES, Adler R, Bilezikian J, et al. The clinical diagnosis of osteoporosis: a position statement from the National Bone Health Alliance Working Group[J]. Osteoporos Int,2014,25(5):1439-1443. DOI: 10.1007/s00198014-2655-z.

[2] Hangartner TN, Warner S, Braillon P, Jankowski L, Shepherd J. The Official Positions of the International Society for Clinical Densitometry: acquisition of dual-energy X-ray absorptiometry body composition and considerations regarding analysis and repeatability of measures[ $\mathrm{J}]$. J Clin Densitom. 2013. 16(4): 520-536. DOI: 10.1016/j.jocd.2013.08.007

[3] Kilic G, Ozgocmen S. Hand bone mass in rheumatoid arthritis: A review of the literature[J]. World J Orthop. 2015. 6(1): 106-116.DOI: 10.5312/wjo.v6. i1. 106

Acknowledgement: none

Disclosure of Interests: None declared

DOI: 10.1136/annrheumdis-2019-eular.631

\section{AB0357 CORRELATION BETWEEN DISEASE ACTIVITY AND MENTAL HEALTH OF RA PATIENTS- ASSESSMENT WITH SMART SYSTEM OF DISEASE MANAGEMENT (SSDM) MOBILE TOOLS}

Bin Wu ${ }^{1}$, Hua Wei ${ }^{2}$, Jianhong $W_{u^{3}}{ }^{\text {, Yi Zhao }}{ }^{4}$, LI Shengguang ${ }^{5}$, LI Fen ${ }^{6}$, Yuhua $\mathrm{Jia}^{7}$, Hui Xiao ${ }^{7}$, Fei Xiao ${ }^{7}$, Hui Song ${ }^{8}$, SSDM Collaboration Group, China. ${ }^{1}$ Chongqing Hospital of Traditional Chinese Medicine, Chongqing, China; ${ }^{2}$ Northern Jiangsu People's Hospital, Yangzhou, China; ${ }^{3}$ Dazhou Central Hospital, Dazhou, China; ${ }^{4}$ Xuanwu Hospital, Capital Medical University, Beijing, China, Beijing, China; ${ }^{5}$ Peking University International Hospital, Beijing, China; ${ }^{6}$ The Second Xiangya Hospital of Central South University, Changsha, China; ${ }^{7}$ Shanghai Gothic Internet Technology Co., Ltd., Shanghai, China; ${ }^{8}$ Beijing Jishuitan Hospital, Beijing, China

Background: WHO survey showed that the prevalence of anxiety and depression in Chinese population and Chinese patients with chronic dis eases were between $3.1 \%-4.2 \%$ and $3.1 \%-7.3 \%$, respectively. DAS28, HAQ, Hospital Anxiety and Depression Scale (HADS) are commonly used to evaluate RA patients' disease activity, physical function and mental health. All of those evaluations were mainly conducted by health professionals with paper questionnaire previously. SSDM is a nove smart disease management tool that allows patients to do self-evaluation on DAS28, HAQ and HADS by mobile terminal.

Objectives: To estimate the prevalence of anxiety and depression in Chinese patients with RA and to analyze the potential association between disease activity and mental health.

Methods: Under the guidance and training by health professionals, RA patients downloaded SSDM and performed self-evaluation bundle of DAS28, HAQ and HADS with SSDM. DAS28 $<=3.2$ and $\mathrm{HAQ}=0$ are criteria for Treat-to-Target (T2T) and normal physical function, HADS score $>=8$ can be diagnosed with anxiety or depression.

Results: From June 2016 to Jan 2019, 2,635 RA patients (461 male 2,174 female) with a mean age of $49.94 \pm 14.23(11-88)$ years and the median disease duration of 25.37 months from 175 hospitals performed bundle self-evaluation for 7,455 times in total. According to the HADS and DAS28 assessment results, the prevalence of anxiety and depression in all patients was $33.30 \%$ and $39.04 \%$ respectively, which was significantly higher than that in the WHO survey in Chinese population and chronic disease patients. The proportion of patients achieved and failed on T2T was $29 \%$ and $71 \%$, respectively. The prevalence of anxiety and 
depression was $20 \%$ and $25 \%$ among T2T achievers; and $31 \%$ and $38 \%$ among T2T failures, respectively $\left(P_{A}<0.05, P_{D}<0.05\right)$.

According to DAS28, the prevalence of anxiety was $19 \%, 20 \%, 27 \%$ and $41 \%$ in Remission (Rem), low disease activity (LDA), moderate disease activity (MDA) and high disease activity (HDA) subgroups respectively, the prevalence of depression was $24 \%, 26 \%, 33 \%$ and $49 \%$ respectively. The correlation coefficients of anxiety (A) and depression (D) with DAS28 were $r_{A}=0.9318$ and $r_{D}=0.9334$, respectively. It suggested that with the increase of disease activity, the proportion of RA patients with anxiety and depression increased significantly.

Combined with physical function evaluation results, the overall prevalence of anxiety (23.65\%) and depression (33.20\%) in the normal HAQ group was significantly lower than that in the abnormal HAQ group (A: $36.37 \%$, D: $\left.43.19 \%, x_{A}^{2}=4.52, x_{D}^{2}=6.21, p_{A}<0.05, p_{D}<0.05\right) .30 .50 \%$ of the patients with $\mathrm{HAQ}=0$ and $\mathrm{DAS} 28<=2.6$ were still depressed. The analysis showed that these subgroup patients comorbid with other rheumatic diseases (SS 37\%, SLE 15\%) or suffer adverse events (abnormal WBC count: 19\%, abnormal liver function: 15\%) during treatment.

Conclusion: Higher prevalence of anxiety and depression were associated with higher levels of disease activity and worse physical function. Rheumatic comorbidity and adverse events (ADEs) were potentially associated with depression in the Rem subgroup patients with normal physical function. SSDM is an effective mobile interface to monitor and study entanglement of disease activity, physical function and mental health in RA patients, which build a foundation for proactive interventions in future. Disclosure of Interests: None declared

DOI: 10.1136/annrheumdis-2019-eular.6787

\section{AB0358 $\quad$ WRIST BONE MINERAL DENSITY AND WRIST SYNOVITIS IN RHEUMATOID ARTHRITIS}

Kaouther Ben Abdelghani ${ }^{1}$, Makhlouf yasmine ${ }^{1}$, Saoussen Miladi ${ }^{1}$, Alia Fazaa ${ }^{1}$, Leila Souebni ${ }^{1}$, Selma Chekili ${ }^{1}$, Kmar Ouenniche $^{1}$, Salma Kassab ${ }^{1}$,

Sonia Kechaou', Zakraoui Leith', Ahmed Laatar'. ' ${ }^{1}$ Mongi Slim Hospital, Tunis, Tunisia; ${ }^{2}$ Institut Pasteur, Tunis, Tunisia

Background: Bone involvement in rheumatoid arthritis is characterized by focal articular bone loss around inflamed small joints of the hands.[1] Therefore, precise quantification of hand bone loss may predict the severity and the progression of the disease.

Objectives: The aim of this study is to find a correlation between wrist bone mineral density (BMD) and sonographic wrist synovitis in patients with rheumatoid arthritis (RA).

Methods: RA patients were examined by bone mineral density (BMD) measurement of the wrist using dual-energy X-ray absorptiometry (DXA) according to standard protocols for positionning and analysis (area measured at a level of $33 \%$ ). Patients were diagnosed as having osteoporosis when the minimum T-score measured was less than -2.5 SD, osteopenia if T-score between $-2,5$ and -1 SD [2]. Ultrasound of the wrist was done by an operator with experience in musculoskeletal ultrasound with an esaote My lab60 machine. The wrist joint (inferior radio-ulnar joint, mediocarpal joint and ulnar recessus) was assessed on dorsal side in Gray and Power Doppler (PDUS) scales. Semi-quantitative score was used to sum the synovitis score.[3]

Statistical analysis comparing synovitis and BMD was performed using Kruskal Wallis Test, a non-parametric test (Mann-Whitney U-test) and chi-square, as appropriate.

Results: The study included 24 RA patients with female predominance (sex ratio $=0,3)$. The mean age was 59.3 [47-71] years-old. The duration of the disease was 10.2 years [0,83 -23] and the body mass index was $27.3(18.97-36.98) \mathrm{kg} / \mathrm{m} 2$. RA was erosive in $75 \%$ of cases. Sixty-seven percent of patients received calcium supplementation. All women experienced already menopause. Most of them (75\%) were on corticosteroids at the average dose of $5 \mathrm{mg}$ [5-15], two of whom were not supplemented. Only one quarter of patients had physical activity. BMD showed that $45 \%$ of patients suffered from osteoporosis and $25 \%$ are osteopenic with a mean T-score of $-1.92[-4.3 ; 1.4]$ and mean bone mass of 0.475 $\mathrm{g} / \mathrm{cm}^{2}[0.239,0.712]$. Forty-eight wrists were assessed by ultrasound. Mediocarpal joint was the most affected in Gray scale and Doppler. Fifty-eight percent of patients had moderate to severe synovitis when combined scoring was used. Twenty nine percent of patients $(29,1 \%)$ had grade 3 at Gray Scale and $25 \%$ had Doppler scale grade 3.The wrist BMD (Tscore) in RA with high disease activity was lower than those with moderate or low disease activity $(-2.21$ vs -2.05 and $-2,21$ vs 0.6 respectively; $p=0,431)$. There was no correlation between BMD of the wrist and the disease duration as well as the activity of the disease DAS28 VS (respectively $p=0.4, p=0.58$ ). The percentages of wrist synovitis in osteoporosis group were significantly higher than in osteopenia group $(81.8 \%$ vs. $66 \%, p=0,03)$. Moroever, Gray scale grade 3 synovitis in osteoportic patients was higher than osteopenic patients $(62,5 \%$ vs $25 \%$ respectively, $\mathrm{p}=0,041)$. Results also showed a correlation between BMD and doppler mode grade 3 (83\%vs 16,7 respectively, $\mathrm{p}=0,014)$.

Conclusion: This study shows a highly significant correlation between the BMD of the wrist and synovitis. Local inflammation of the wrist is an important factor of local bone loss that should be acted upon in order to avoid fractures, especially Colles' fracture.

\section{REFERENCES}

[1] Hansen M,et al. Bone loss in rheumatoid arthritis.. Scand J Rheumatol. 1996;25:367-376

[2] World Health Organization (2004) WHO scientific group on the assessment of osteoporosis at primary health care level.p. 5-7.

[3] D'Agostino M L, et al.Scoring ultrasound synovitis in rheumatoid arthritis a EULAR-OMERACT ultrasound taskforce-Part 1: definition and development of a standardised, consensus-based scoring system.RMD Open 2017;000428.

Disclosure of Interests: None declared

DOI: 10.1136/annrheumdis-2019-eular.6986

\section{AB0359 TREATING STRATEGY FOR ELDERLY RHEUMATOID ARTHRITIS PATIENT, ESPECIALLY WHOSE AGE IS MORE THAN 75}

Ichiro Yoshii ${ }^{1}$, Tatsumi Chijiwa ${ }^{2}$, Naoya Sawada ${ }^{3}{ }^{1}$ Yoshii Hospital, Rheumatology and Musculoskeletal Medicines, Shimanto City, Japan; ${ }^{2}$ Kochi Memorial Hospital, Rheumatology, Kochi, Japan; ${ }^{3}$ Doha Onsen Hospital, Rheumatology, Matsuyama, Japan

Background: A population of elderly rheumatoid arthritis (ERA) is increas ing, probably due to treatment developed and simply elderly population increase. In aging, functional activity in daily life, immunity, especially $T$ cell function, and neural response decline and deteriorations become manifested. Treatment must not be same as that of young patient. Objectives: To evaluate our treatment method and strategy for ERA

Methods: From August 2010 to July 2015, 576 patient who have been treated in the institute continuously for more than 3 years were referred. In these, patients were classified in according with age at baseline (BL); younger than 65 (G-Y), from 65 to 74 (G-O), and no less than 75 (GOO). Mean 28-joints disease activity score (DAS28), Health Assessment Questionnaire Disability Index (HAQ), Pain Score with visual analog scale (PS-VAS), drug administration history and dosage, were recorded.

For ERA, we have adopted a treating strategy called "Touch Down Strategy", what configures three tactics; 1) From BL, methotrexate (MTX $6 \mathrm{mg} /$ week or tacrolimus (TAC) $1.5 \mathrm{mg} /$ day administer. 2) Increase or maintain drug dosage until clinical remission is attained or start bDMARDs when remission is not attained in 3 months, and in case, glucocorticoid (GCS) administered with every other month interval. 3)When clinical remission is attained, GCS tapering started immediately and csDMARDs tapering considered. Tapering of bDMARDs is the last order.

ERA patients were treated under these tactics. Monitored DAS28, HAQ score and PS-VAS were calculated for each group and compared with ANOVA with Bonferroni correction.

Results: $\mathrm{HAQ}$ at baseline demonstrated significantly higher in G-OO than the other groups. Prevalence of DAS28 remission were $76.4 \%, 89.6 \%$, and $87.2 \%$, while mean length from BL to DAS28 remission was $2.9,2.5$ and 4.0 months for G-Y, G-O, and G-OO, respectively. bDMARDs administration ratio was $19.8 \%, 20.6 \%$, and $18.0 \%$, while mean MTX dosage was $8.6 \mathrm{mg}$, $8.6 \mathrm{mg}$, and $7.4 \mathrm{mg} /$ week, for G-Y, G-O, and G-OO respectively. GCS administration ratio and mean dosage until DAS28 remission were $24.2 \%$ and $2.96 \mathrm{mg}, 38.0 \%$ and $2.41 \mathrm{mg}$, and $42.6 \%$ and $2.71 \mathrm{mg} /$ day, while after remission $19.3 \%$ and $5.68 \mathrm{mg}, 21.1 \%$ and $4.58 \mathrm{mg}$, and $26.4 \%$ and $2.14 \mathrm{mg} /$ day, for G-Y, G-O, and G-OO, respectively (Table). Conclusion: Our Touch Down Strategy can work effective for ERA, especially for elderly ERA whose age is over 75 years old. Just by doing take care for risk of comorbidities, ERA can be well controlled their disease activity. 